\title{
Pipe Diameter Optimization using Genetic Algorithm- A Case Study
}

\author{
M.P.P.Dhanaseela and T.Tingsanchali
}

\begin{abstract}
A systematic study on the application of genetic algorithm (GA) in pipe diameter optimization was carried out. The aim of the study was to develop a genetic based programme and apply it to a case study, distribution system of Weligama water supply scheme. Then the performance of Genetic algorithm was compared with non linear programming (NLP).

It was found that the genetic algorithm method using the standard three operators is particularly effective in finding global optimal solution for the case study network. It should be noted that the genetic algorithm technique is ideally suited to discrete problems such as selection of commercially availably pipe sizes.
\end{abstract}

Keywords: Genetic Algorithm, Global Optimum, Pipe Network

\section{Introduction}

Optimization concept is intrinsically tied to human intuition. A desire for perfection or a search for the best is naturally demanded. As a vital part of the water supply systems, water distribution networks represent one of the largest infrastructure assets of water industrial society. In water supply design point of view, whether the ultimate goal is to assure for more reliable supply of water with respect to space and time, the achievements of economic advantage are more meaningful, because funds available for the development of new infrastructure become increasingly scarce. As a result, pipe network optimization becomes a field of study in its own right.

Genetic algorithm is proposed as one possibility of optimum search algorithms. Since the early development by HOLLAND in 1975[4], the algorithms have been theoretically and empirically proved to be a very effective and robust search method. Advantages of genetic algorithm are due to its simplicity, straightforwardness and commitment for better and better solutions. Genetic algorithms were employed in a wide-ranging of pipe network optimization problems and the capability of genetic algorithms were evident in various research studies [7], [8], [12].

\section{Literature Review}

Golberg [2] examined the mechanics, power and application of a genetic algorithm to a steady state optimization of a serial liquid pipeline. The objective was to minimize power, while supplying a specified flow and maintaining allowable pressures. Simple three operator genetic algorithm was applied to the on-off control of 40 pumps in 10 pump stations along a serial pipeline. The pipeline model has been programmed in Pascal. Constraints have been adjoined to the problem with an exterior penalty function. The genetic algorithm was run with 100 population size. The probability of crossover and probability of mutation were taken as 0.7 and 0.01 . It was found that GA gives near optimal operation pump schedules quickly.

Savic and Walters [7] described the development of the computer model GANET for the least cost design of water distribution networks and further more demonstrated the superior capability of GA against other optimization techniques. Least cost pipe network design was applied to a sample network with a single source at a $210 \mathrm{~m}$ fixed head and eight pipes arranged in two loops. A binary string made of eight sub strings was used for coding the problem.

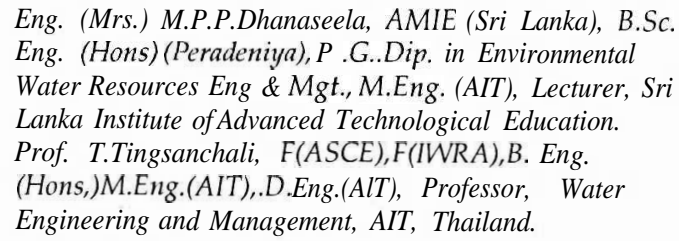


The evaluation function determined the cost of a solution. Rank selection was used for reproduction. Ultimate results showed that the GA produces good design.

Meier [5] described the use of a GA to optimize the sampling design. GA was applied to a network model for a small town in Ohio. Probability of crossover and mutation was taken as 0.7 and 0.02 respectively. GA was shown to perform extremely well, matching the optimal solution produced by complete enumeration in a series of validation tests.

\section{Study Area}

GA is applied to a water supply pipe network in Weligama area in Matara district which has been constructed in 1991. This water supply scheme covers about $13 \mathrm{~km}^{2}$ area of Matara District in Southern province. Designed demand is $10,950 \mathrm{~m}^{3} /$ day by 2011 . In this study, only the distribution system is analysed, where the water flows under gravity from a reservoir of $910 \mathrm{~m}^{3}$ capacity at Kottegoda. It consists of pipe lines of PVC Type 600 of several diameters ranging from $50-250 \mathrm{~mm}$. Information on Elevations, pipe lengths, and demand were collected.

\section{Methodology}

\subsection{Theoretical background-Genetic} Algorithms

Genetic algorithms are search algorithms based on Darwin's theory of survival of the fittest [2] Through the three basic operations; reproduction, crossover and mutation, genetic algorithms search for optimum solution. Decision variables should be represented in a appropriate coding scheme. The search begins with this group of strings. Every string in a population is processed concurrently generation-by-generation under controlling of genetic operations. Reproduction operator selects the effective strings from population relying on their objective functions or fitness values. Effective strings are then controlled to breed for new generation by crossover. Hence, new strings are introduced by gathering information from fittest pairs of string or chosen mate. Mutation operator simulates a natural adaptation by transmuting some bits in string to prevent irrecoverable losses of data. Penalty function is introduced to prevent searching in constrained violating domain.
Termination criteria are predefined to stop the search algorithms.

\subsection{Pipe network Optimization}

Water distribution network optimization is a procedure to seek for design variables of a network to its goal of optimality, within the constraints. Objective function or the cost function should be formulated whose least values is searched in an optimization procedure.

\subsubsection{General pipe network Optimization Problem}

Objective function of the general pipe network optimization problem can be shown as;

Minimize $\mathrm{F}(\mathrm{D})=\sum_{i=1}^{N} c\left(D_{i}, L_{i}\right)$

Where $c\left(D_{i}, L_{i}\right)$ is the cost of the pipe with the diameter $D_{i}$ and the length $\mathrm{L}$, and $N$ is the total number of pipes in the system.

This objective function is minimized under the flowing constraints:

For each junction,

$$
\sum Q_{\text {in }}-\sum Q_{\text {out }}-\sum Q_{e}=0
$$

$Q_{\text {in }} \cdot Q_{\text {out }}$ and $\boldsymbol{Q e}$ are $_{\mathbf{f l}^{\circ \mathrm{w}}}$ into the junction, oUt of the junction and external inflow or demand at the junction node respectively.

Where $Q_{\text {in }}, Q_{\text {out }}$ and $Q_{e}$ are flow into the junction, out of the junction and external inflow or demand at the junction node respectively.

For each loop,

$$
\sum h_{f}-\sum E_{p}=0
$$

Where $h$, and $E$, are head loss and energy put into the liquid by a pump.

For each node,

$$
H^{\max }>H_{j}>H^{\min } \quad ; \mathrm{j}=1, \ldots . \mathrm{n}
$$


Where $H_{j}, H^{\max }$ and $H^{\text {min }}$ are head at node j, maximum and minimum allowable head respectively.

For each pipe,

$$
V^{\max } \geq V_{j} \geq V^{\min } \quad ; \mathrm{j}=1, \ldots . \mathrm{n}
$$

Where $V_{j}, V^{\max }$ and $V^{\min }$ are velocity at pipe $\mathrm{j}$, maximum and minimum allowable flow velocity respectively.

\subsection{Computer Program of Three Operator Genetic algorithm}

Microsoft visual $\mathrm{C}++$ version 6.0 was used and the programme was written in $\mathrm{C}$ language. Elevation, nodal demand, available pipe diameter sizes and their unit prices were the input data files.

Binary coding is used to code the decision variable. GA generates the initial population of solutions (of say, size $N=100$ ) using a random number generator. Number of population

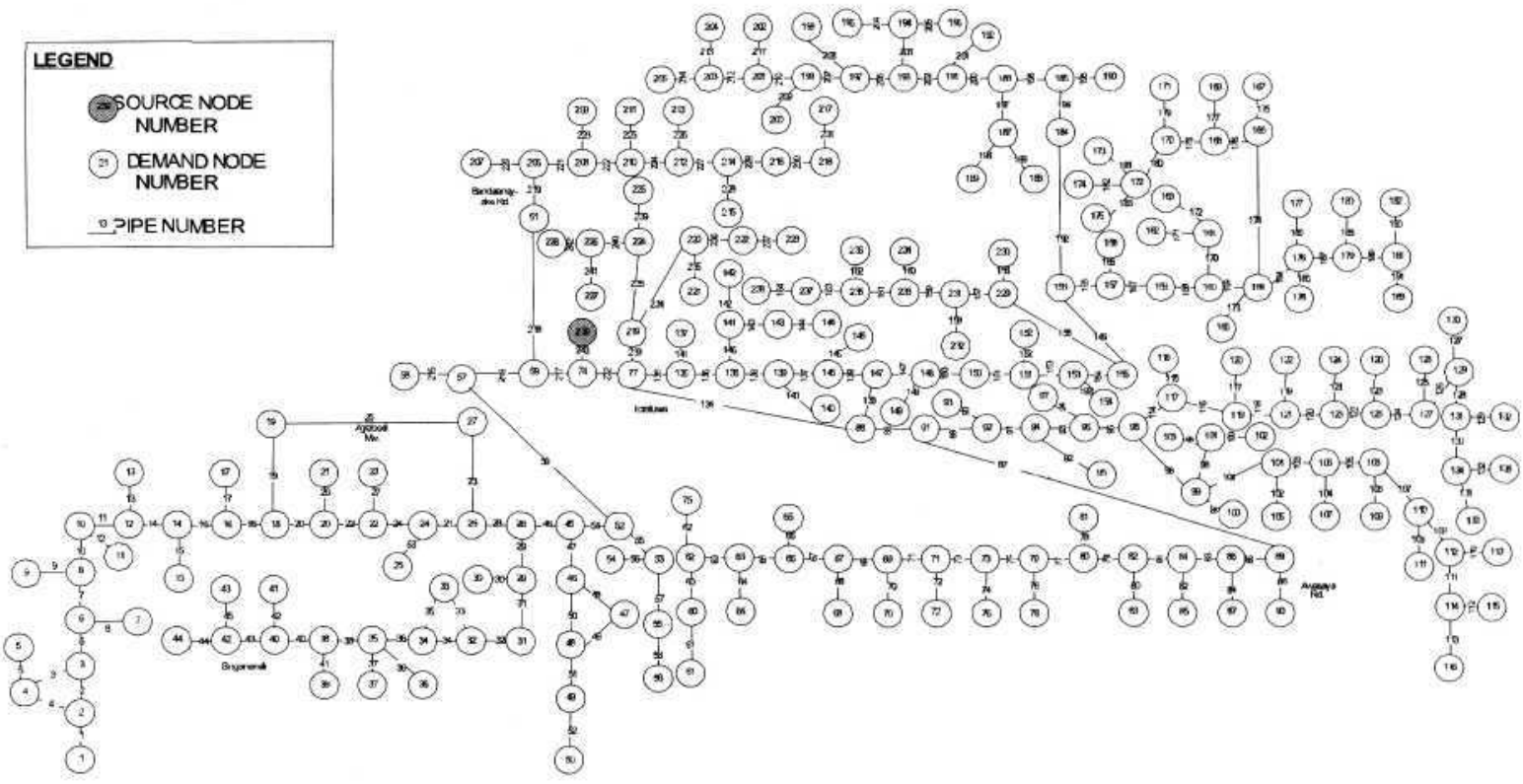

Figure 1- Layout of the pipe network

\section{Analysis}

\subsection{Assumptions and design criteria}

The following assumptions were made in hydraulic design.

- The minimum service pressure head in the distribution system should not be less than $10 \mathrm{~m}$ and the maximum service pressure head should not higher than $50 \mathrm{~m}$.

- The Hazen Williams coefficient $\mathrm{C}$ is assumed to be 130 .

- Minimum and maximum flow velocities are taken as $0.6 \mathrm{~m} / \mathrm{s}$ and $1.6 \mathrm{~m} /$ s respectively.

- The flow state is assumed to be steady for the whole network.

- The maximum head loss allowance should not exceed 10 meters per kilometre length of pipe line. should be in the range of 30-200[3]. Options available for existing pipes are arranged in terms of increasing cost and the binary string representation of available pipe sizes is given in the Table 1.

To evaluate the fitness of a string, the string must be converted into a design solution by mapping a string value onto a variable value. Assume one bit of one substring for one variable is represented as $a_{1}, a_{2} \ldots a_{n}$, the value of the substring (corresponding diameter index) can be calculated as

$\mathrm{S}_{\mathrm{i}}=\sum_{n=1}^{N b i t} a_{n} b^{(N b i t-n)}$

Where Nbit is the number of bits in the substring and $b$ is equal to 2 . 
Table 1- Binary string representation, corresponding diameter index of available pipe sizes and unit prices

\begin{tabular}{|c|c|c|c|}
\hline $\begin{array}{c}\text { Binary } \\
\text { substring } \\
\text { coding } \\
\text { pipe size }\end{array}$ & $\begin{array}{c}\text { Dia. } \\
\text { index }\end{array}$ & $\begin{array}{c}\text { Available } \\
\text { pipe } \\
\text { diameters } \\
(\mathrm{mm})\end{array}$ & $\begin{array}{c}\text { Unit price } \\
(\$ / \mathrm{m})\end{array}$ \\
\hline 000 & 0 & 47 & 0.45 \\
\hline 001 & 1 & 58 & 0.70 \\
\hline 010 & 2 & 69 & 0.92 \\
\hline 011 & 3 & 83 & 1.30 \\
\hline 100 & 4 & 102 & 1.95 \\
\hline 101 & 5 & 148 & 4.10 \\
\hline 110 & 6 & 208 & 9.00 \\
\hline 111 & 7 & 280 & 13.00 \\
\hline
\end{tabular}

Network cost is calculated for each population by taking the summation of product of pipe length and unit cost of pipe.

Two governing equations used are continuity equation and Hazen William's head loss equation [9].

$\mathrm{hf}=\underline{10.675 L_{i} * Q_{i} *\left|Q_{l}\right|^{0.852}}$

$$
C_{i}^{1.852} * D_{i}^{4.8704}
$$

Where

$L=$ Length of the pipe

$D_{i}-$ Diameter of the pipe

$Q_{i}=$ Flow through the pipe

$C_{i}=$ Hazen William coefficient

The head loss equation is expressed for each pipe and the continuity equation is applied at each node throughout the water supply distribution system. The flow and head loss conditions are determined for each pipe in the loops by solving the two equations iteratively.

Knowing the flow rate through each pipe, velocities can be calculated.

$$
V_{i}=\frac{Q_{i}}{\pi\left(D_{i}^{2} / 4\right)}
$$

Knowing the head loss and elevation at each node, pressure head can be calculated.

The total penalty cost is taken as the summation of the product of the pressure head constraint violations $\left(\mathrm{P}_{1}\right)$ times a specified penalty multiplier $\left(R_{1}\right)$ and the product of the velocity constraint violations $\left(\mathrm{P}_{2}\right)$ times a specified penalty multiplier $\left(R_{2}\right) \cdot R_{1}$ is a measure of the worth per metre attributed to pressure heads below and above the allowable minimum and maximum pressure heads. $R_{2}$ is a measure of the worth per metre attributed to velocities below and above the allowable minimum and maximum velocities.

Fitness of each population is calculated as the summation of network cost and the total penalty cost [8].

$f_{i}=F(D)+R_{1} P_{1}+R_{2} P_{2}$

Where $\mathrm{F}(\mathrm{D})=\sum_{i=1}^{N} c\left(D_{i}, L_{i}\right), \mathrm{c}\left(\mathrm{D}_{\mathrm{i}}, \mathrm{L}_{\mathrm{i}}\right)$ is the cost of the pipe with the diameter $D_{i}$ and the length $L i$, and $\mathrm{N}$ is the total number of pipes in the system.

Then the GA generated new members of the new generation by tournament selection method, where two or more chromosomes are randomly picked from the population, and the best one selected for further genetic processing.

The uniform crossover operator is practiced next. It is the partial exchange of bits between two parent strings to form two offspring strings. A crossover probability $\left(p_{c}\right)$ is assumed between (0.5-1.0). The GA randomly picks two strings from the new population. A uniformly distributed random number is then generated in the range 0.0 and 1.0. The GA applies the crossover operator if the random number is less than the probability of crossover.

To ensure no important genetic material is lost, Mutation operator is applied. A probability of mutation $\left(\mathrm{p}_{\mathrm{m}}\right)$ is assumed between 0.01-0.1. The GA considers each string bit by bit in the new generation formed as a result of reproduction and crossover. A uniformly distributed random number in the range of 0.01 to 0.1 is generated. GA applies the mutation operator if the random number is less than the probability of mutation. Otherwise the GA does not apply mutation to that particular bit. The mutation operator changes the value of the bit to the opposite value. (i.e. a 0 to 1 or a 1 to 0 ). This procedure repeats until the optimal value is reached.This solution is tested with the Loop hydraulic simulation model. 


\begin{tabular}{|c|c|}
\hline Random number seed & Least cost $(\mathrm{M} \$)$ \\
\hline 0 & 0.123 \\
\hline 100 & 0123 \\
\hline 500 & 0.123 \\
\hline 1000 & 0.144 \\
\hline 5000 & 0.123 \\
\hline
\end{tabular}

\subsection{Non-linear programming.}

The case study problem is solved using the nonlinear optimization package, "GAMS". The General Algebraic Modelling System (GAMS) language is formally similar to commonly used programming languages. GAMS analysis is carried out independently of genetic algorithm analysis.

A cost function for pipes is fitted (Figure 2) and it is given below (Equation 10).

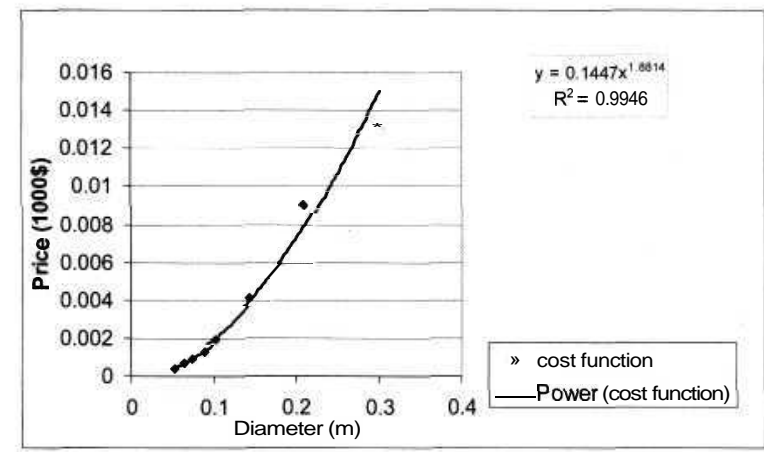

Figure 2 - cost function

$$
=0.144 D^{1.8814} \quad C
$$

Where $C=$ cost of pipes

$\mathrm{D}=$ diameter of pipe in $\mathrm{m}$

Constraints, same as (2)-(5) were included in the model.

\section{Results and Discussion}

\subsection{Searching of global optimality}

Five runs for the case study network problem using different random number seeds were carried out to check whether the global optimality is achieved. A maximum of 50,000 functional evaluations were permitted for each run. The lowest cost achieved in each run is presented in table 2 . The least cost was found at the first of 300 generation.
Table 2- Results of genetic algorithm runs for 10,000 evaluations

\subsection{Parameterselection}

Twenty additional runs were performed to assess the sensitivity of the technique to parameter settings. The quality of solution obtained from GA depends upon several important parameters, such as, population size, probability of crossover $\left(p_{c}\right)$ and probability of mutation $\left(\mathrm{p}_{\mathrm{m}}\right)$. Each parameter was examined during testing process, in the way that only the testing parameter was changed while the others were kept constant. Table 3 shows the GA parameters and lowest cost results.

\section{Table 3-GA Parameters and Cost Results}

\begin{tabular}{|l|c|c|c|c|}
\hline $\begin{array}{l}\text { Run } \\
\text { no. }\end{array}$ & $\begin{array}{l}\text { Pop. } \\
\text { size } \\
(\mathbf{n})\end{array}$ & $\begin{array}{l}\text { Prob. of } \\
\text { crossover } \\
\left(\mathrm{pe}_{\mathrm{c}}\right.\end{array}$ & $\begin{array}{l}\text { Prob. of } \\
\text { mutation } \\
\left(\mathrm{p}_{\mathrm{m}}\right)\end{array}$ & $\begin{array}{l}\text { Lowest } \\
\text { cost } \\
(\mathrm{M} \$)\end{array}$ \\
\hline 1 & 30 & 0.7 & 0.04 & 0.168 \\
\hline 2 & 50 & 0.7 & 0.04 & 0.168 \\
\hline 3 & 100 & 0.7 & 0.04 & 0.144 \\
\hline 4 & 200 & 0.7 & 0.04 & 0.123 \\
\hline 5 & 250 & 0.7 & 0.04 & 0.131 \\
\hline 6 & 300 & 0.7 & 0.04 & 0.168 \\
\hline 7 & 200 & 0.7 & 0.01 & 0.152 \\
\hline 8 & 200 & 0.7 & 0.02 & 0.152 \\
\hline 9 & 200 & 0.7 & 0.03 & 0.152 \\
\hline 10 & 200 & 0.7 & 0.04 & 0.123 \\
\hline 11 & 200 & 0.7 & 0.05 & 0.152 \\
\hline 12 & 200 & 0.5 & 0.04 & 0.123 \\
\hline 13 & 200 & 0.7 & 0.04 & 0.123 \\
\hline 14 & 200 & 1.0 & 0.04 & 0.123 \\
\hline
\end{tabular}

\subsubsection{Effect of Population Size}

Several population sizes were examined. The best number for the population size is taken as 200 , since it gives the lowest cost.

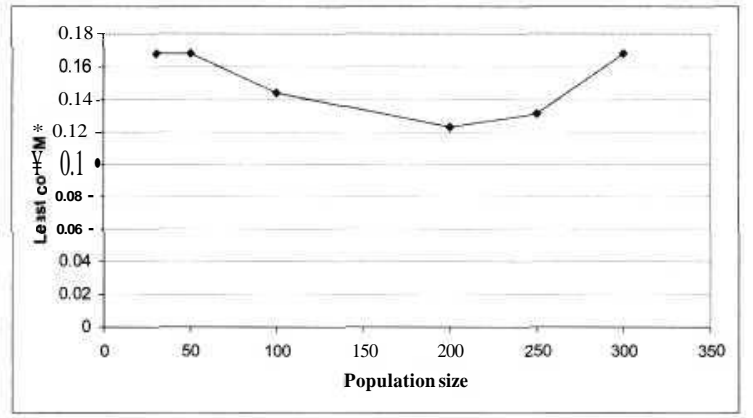

Figure 3- The effect of population size 


\subsubsection{Effect of Probability of Crossover}

The probabilities of crossover of $0.5,0.7$, and 1.0 were examined and the effect is shown in the Figure 4. It shows no effect of probability of cross over.

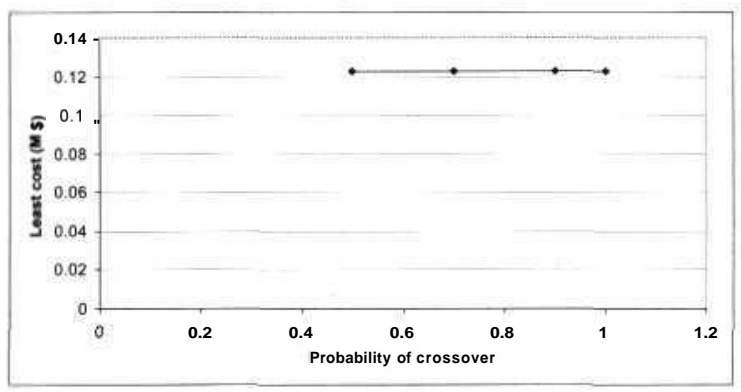

Figure 4-The Effect of Probability of Crossover

\subsubsection{Effect of Probability of Mutation}

Several probabilities of mutation were examined and the effect is shown in the Figure 5 . The best value for the probability of mutation was taken as 0.04 ,since it gives the minimum cost.

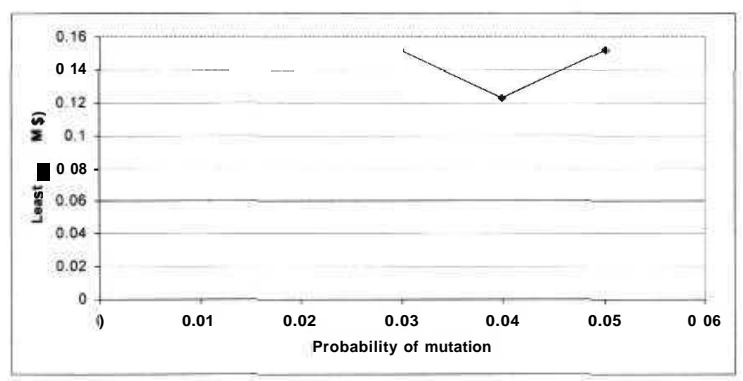

Figure 5-The Effect of Probability of Mutation

\subsubsection{Effect of Penalty Multipliers}

The effect of Penalty multipliers, $R_{1}$ and $R_{2}$ were tested and their effect on the fitness is shown in figure 6 and 7.

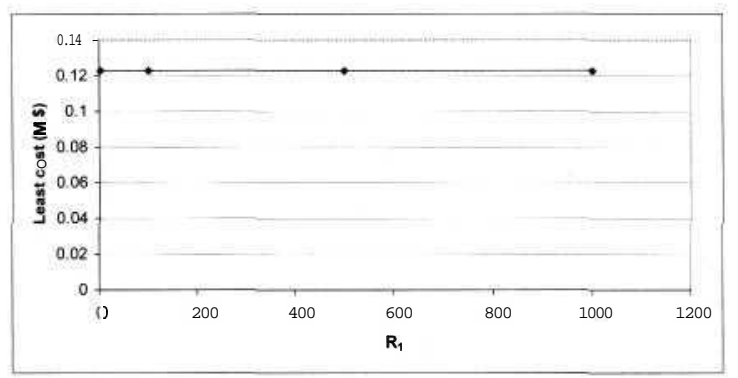

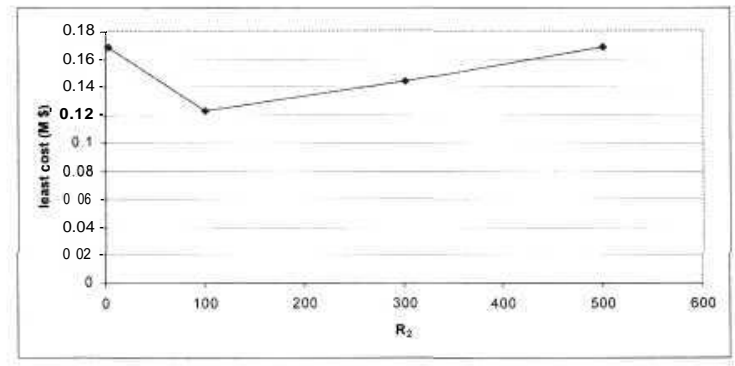

Figure 7 - The effect of $\mathbf{R}_{2}$

\subsection{Final Result of GA}

The run characteristics, criteria formulation for GA and the final result of GA are given in Table 4. GA run took 270 CPU min on a Pentium 4, CPU $1600 \mathrm{MHz}$, RAM 130,544 KB computer to search the least cost of the pipe network as Million US \$ 0.123. Optimum diameter given by the GA are shown in Table 5.

The solution from the GA was simulated with the help of LOOP hydraulic simulation model.

Table 4- Results of GA

\begin{tabular}{|l|c|}
\hline \multicolumn{1}{|c|}{ Item } & Results \\
\hline Population size & 200 \\
\hline $\begin{array}{l}\text { Execution time in } \\
\text { minuets with Pentium } \\
\text { IV } \\
1600 \mathrm{Mhz}, \text { Ram } \\
130544 \mathrm{~kb}\end{array}$ \\
\hline $\begin{array}{l}\text { Number of alleles per } \\
\text { gene }\end{array}$ \\
\hline Length of chromosome \\
\hline $\begin{array}{l}\text { Probability } \\
\text { crossover }\end{array}$ & 270 \\
\hline Probability of mutation & $0.7243)$ \\
\hline Type of selection & Tournament selection \\
\hline Type cross of over & Uniform crossover \\
\hline Type of mutation & Uniform mutation \\
\hline Best fitness (M $\$)$ & 0.123 \\
\hline
\end{tabular}

\subsubsection{Result of Non Linear Program}

It gave the least cost as $\mathrm{M} \$ 0.11$. The optimization run took 3.5CPU min on a Pentium 4, CPU $1600 \mathrm{MHz}$, RAM 130,544 KB computer. The solutions given by NLP are shown in Table 5. Many of the values given as pipe diameters could not found in the market. Therefore it is needed to round up the pipe sizes to the nearest available diameter manually and run Loop software to ensure the rounded solution satisfies the constraints. Then the result came similar to that of genetic algorithm.

Figure 6 - The effect of $R_{1}$ 

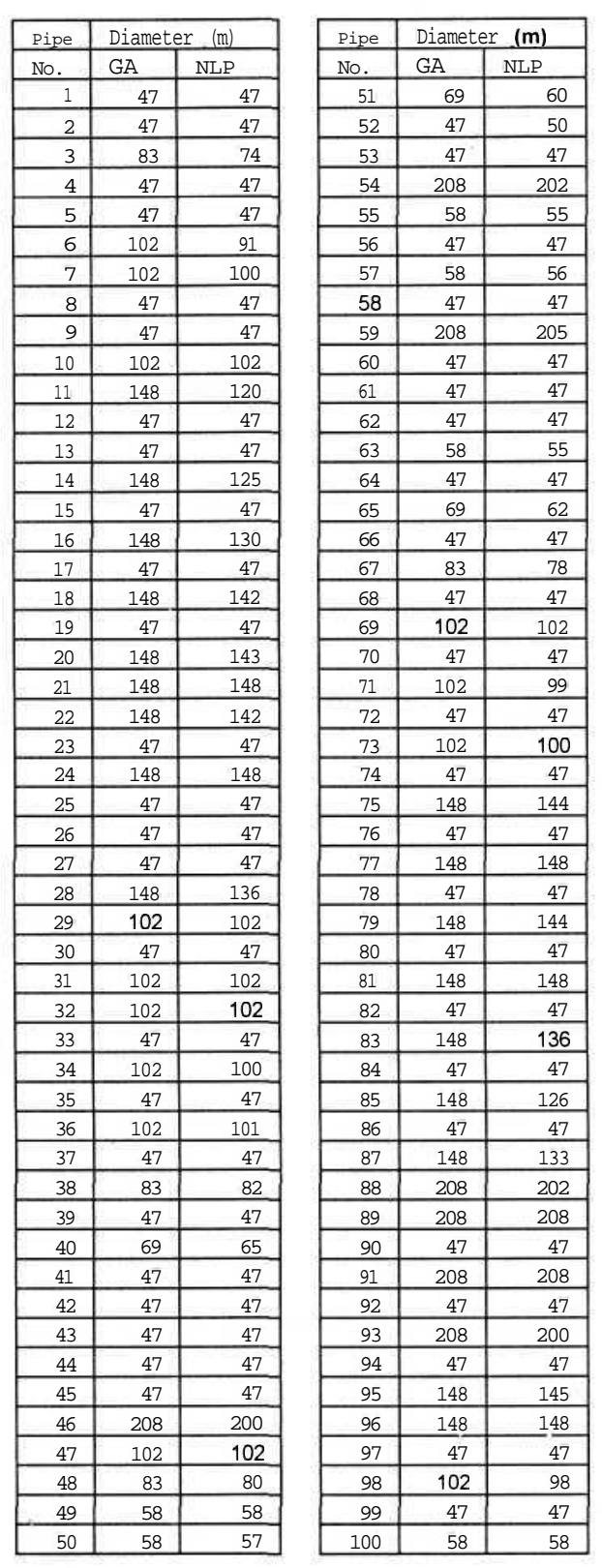

\section{Conclusions}

Non linear optimization is the fastest of the two optimization techniques applied to the case study network. But one needs to fit a cost function to the discrete pipe cost data and this may leads to inaccuracies. On the other hand it is needed to round the continuous solution found either up or down to the nearest discrete pipe sizes manually. Additional computer runs are needed in Loop software to ensure that the rounded solution actually satisfies pressure and velocity constraints. Therefore, although non linear optimization is quick, reaching to a required discrete pipe solution takes days.

\begin{tabular}{|c|c|c|}
\hline Pipe & \multicolumn{2}{|c|}{ Diameter (m) } \\
\hline No. & $\mathrm{GA}$ & NLP \\
\hline 151 & 208 & 208 \\
\hline 152 & 47 & 47 \\
\hline 153 & 208 & 208 \\
\hline 154 & 208 & 47 \\
\hline 155 & 102 & 199 \\
\hline 156 & 58 & 102 \\
\hline 157 & 83 & 83 \\
\hline 158 & 47 & 47 \\
\hline 159 & 69 & 67 \\
\hline 160 & 47 & 47 \\
\hline 161 & 58 & 56 \\
\hline 162 & 47 & 47 \\
\hline 163 & 47 & 47 \\
\hline 164 & 47 & 47 \\
\hline 165 & 148 & 133 \\
\hline 166 & 47 & 47 \\
\hline 167 & 148 & 126 \\
\hline 168 & 148 & 146 \\
\hline 169 & 148 & 145 \\
\hline 170 & 47 & 47 \\
\hline 171 & 47 & 47 \\
\hline 172 & 47 & 47 \\
\hline 173 & 47 & 47 \\
\hline 174 & 102 & 100 \\
\hline 175 & 47 & 47 \\
\hline 176 & 83 & 81 \\
\hline 177 & 47 & 47 \\
\hline 178 & 69 & 65 \\
\hline 179 & 47 & 47 \\
\hline 180 & 47 & 47 \\
\hline 181 & 47 & 47 \\
\hline 182 & 47 & 47 \\
\hline 183 & 47 & 47 \\
\hline 184 & 83 & 81 \\
\hline 185 & 47 & 47 \\
\hline 186 & 47 & 47 \\
\hline 187 & 47 & 47 \\
\hline 188 & 47 & 47 \\
\hline 189 & 47 & 47 \\
\hline 190 & 47 & 47 \\
\hline 191 & 47 & 47 \\
\hline 192 & 148 & 140 \\
\hline 193 & 47 & 47 \\
\hline 194 & 148 & 140 \\
\hline 195 & 47 & 47 \\
\hline 196 & 148 & 136 \\
\hline 197 & 69 & 66 \\
\hline 198 & 58 & 58 \\
\hline 199 & 47 & 47 \\
\hline 200 & 148 & 136 \\
\hline
\end{tabular}

\begin{tabular}{|r|r|r|}
\hline Pipe & \multicolumn{2}{|c|}{ Diameter } \\
\hline No. & \multicolumn{1}{|c|}{ GA } & \multicolumn{1}{|c|}{ NLP } \\
\hline 201 & 47 & 47 \\
\hline 202 & 148 & 148 \\
\hline 203 & 69 & 67 \\
\hline 204 & 47 & 47 \\
\hline 205 & 47 & 47 \\
\hline 206 & 148 & 146 \\
\hline 207 & 102 & 102 \\
\hline 208 & 69 & 69 \\
\hline 209 & 47 & 47 \\
\hline 210 & 102 & 101 \\
\hline 211 & 47 & 47 \\
\hline 212 & 83 & 82 \\
\hline 213 & 47 & 47 \\
\hline 214 & 69 & 65 \\
\hline 215 & 47 & 47 \\
\hline 216 & 208 & 179 \\
\hline 217 & 208 & 208 \\
\hline 218 & 83 & 78 \\
\hline 219 & 83 & 79 \\
\hline 220 & 47 & 47 \\
\hline 221 & 83 & 83 \\
\hline 222 & 69 & 69 \\
\hline 223 & 47 & 47 \\
\hline 224 & 69 & 64 \\
\hline 225 & 47 & 47 \\
\hline 226 & 47 & 47 \\
\hline 227 & 58 & 51 \\
\hline 228 & 47 & 47 \\
\hline 229 & 58 & 52 \\
\hline 230 & 47 & 47 \\
\hline 231 & 47 & 47 \\
\hline 232 & 280 & 262 \\
\hline 233 & 102 & 102 \\
\hline 234 & 69 & 67 \\
\hline 235 & 47 & 47 \\
\hline 236 & 47 & 47 \\
\hline 237 & 47 & 47 \\
\hline 238 & 83 & 77 \\
\hline 239 & 47 & 47 \\
\hline 240 & 69 & 66 \\
\hline 241 & 47 & 47 \\
\hline 242 & 47 & 47 \\
\hline 243 & 280 & 280 \\
\hline & & \\
\hline
\end{tabular}

The computer time required in genetic algorithm is comparatively long. It is found that the genetic algorithm method using the standard three operators is effective in finding the optimal solution, especially discrete problem like finding the optimum pipe diameters, where commercially available pipe sizes are discrete.

\section{References}

1. Brooke, A., Kendrick, D., and Meeraus, A. GAMS a user's guide. http://www.gams.com, Visited, $5^{\text {th }}$ January 2004.

2. Goldberg, D.E. and Kou, C.H., Genetic Algorithms in Pipeline Optimization, J. Aerospace Engineering, Vol. 1, No. 2,pp 128141,April 1987. 
3. Goldberg, D.E., Genetic Algorithms in Search, Optimization, and Machine Learning, AddisonWesley Publishing, 1989.

4, I lolland, J.H., Genetic Algorithms and The Optimal allocation of Trials, SIAM J. on Computing, Vol. 2, No. 2, ,pp 88-105, June 1973.

5. Kampanad, B., the development of a Genetic Algorithm for real time water allocation and water scheduling in complex irrigation systems. A thesis submitted for the degree of Doctor of philosophy, the University of Edinburgh, 2001.

6. Meier, W., Sampling design for network model calibration using genetic algorithms, J. water resources planning and management, ASCE, Vol.126.No.4,pp.245-250, 2000.

7. Reis L. and Chaudhry, Optimal location of control valves in pipe networks by genetic algorithm. J. Water Resour. Mgt.,. Vol.123, No.6,pp.317-320, 1997.

8. Savic,D.A., and Walters, G.A, Genetic Algorithm for least cost design of water distribution networks, J. water res. planning and mgt. , ASCE, Vol.123.No.2,pp.67-77, 1997.

9. Simpson, A.R, Genetic Algorithms compared to other techniques for pipe optimizations, J. water res. planning and mgt., ASCE, Vol.120,No.4,pp.423-443, 1994.

10. Streeter, V.L. and Wylie, E.B.,Fluid Mechanics, $6^{\text {th }}$ ed.,, McGraw-Hill, U.S.A. ,1975.

11. Thawatgau T. and Anat A., Bangkok Metropolitan Water Distribution System Analysis. Research report submitted to Asian Institute of Technology, Bangkok, Thailand. ,1999.

12. Vuuren van,S.J., Application of genetic algorithms - detemination of the optimal pipe diameters, J. Water SA, Vol.28.No.2,pp.217226,2002 .

13. Wu.Z.Y. and Simpson A.R., Competent Genetic-Evolutionary Optimization Of Water Distribution systems. J. Computing in Civil Engineering, Vol.15 No.2, pp. 89-101, 2001. 